\title{
Credibilidad del testimonio de los menores en función de la edad
}

\author{
Credibility of children's testimony according to age \\ Dolores Padilla-Racero \\ Universidad de Málaga, España
}

\begin{abstract}
Resumen
En las rupturas de parejas con hijos, el juzgado ha de determinar el régimen de comunicación del menor con el progenitor que no tiene su guarda y custodia. El testimonio del menor es un elemento controvertido, especialmente si existen denuncias de maltrato o abuso sexual. La escuela de Gardner defiende que el niño es mentiroso por naturaleza, sin embargo autores como Clemente y Padilla (2015) demuestran que los niños tienden a decir la verdad. Partiendo de ello, este estudio analiza si la edad de los menores es una variable mediadora en la veracidad del testimonio y en su posible manipulación.
\end{abstract}

Palabras clave: manipulación, síndrome de alienación parental, testimonio, edad, menores.

\begin{abstract}
In the breaks of couples with children, the court must determine the communication system of the child with the parent who does not have his custody. The testimony of minors is a controversial element, especially if there are allegations of abuse or sexual abuse. School Gardner argues that the child is a liar by nature, but authors like Clement and Padilla (2015) show that children tend to tell the truth. On this basis, this study examines whether the age of the children is a mediating variable in the truthfulness of the testimony and its possible manipulation
\end{abstract}

Keywords: manipulation, parental alienation syndrome, testimony, age, minor.

\section{Justificación teórica}

El establecimiento del tiempo de comunicación de los hijos con el progenitor que no ostenta la guarda y custodia, es algo que, en ausencia de acuerdo entre ambos progenitores, va a decidir el órgano judicial competente, desde el nacimiento del menor, hasta la mayoría de edad de éste. El juez del órgano judicial donde se sustenta el proceso de separación o divorcio de la pareja con hijos, puede recabar información directamente del menor o hacerlo a través de los informes de los equipos psicosociales o equipos técnicos adscritos al juzgado dentro de su labor de auxilio del órgano judicial. El alcance del testimonio del menor, va a depender del concepto de credibilidad del menor que estas entidades implicadas tengan. Si hablamos de la credibilidad del testimonio de los menores, dentro de los procedimientos judiciales de separación o divorcio, donde se dilucida con que progenitor van a convivir éstos y el régimen de comunicación con el progenitor con el que no van a convivir, tenemos que hablar del SAP, constructo formulado por el psiquiatra estadounidense Richard Gardner (1985). Vaya por delante, que a pesar de carecer de toda cientificidad el concepto, su aplicación como tal, es un hecho en el ámbito judicial. Gardner (1991, p.15) define este síndrome como: "Trastorno infantil que surge casi exclusivamente en el contexto de las disputas por la custodia de los niños. Su manifestación primaria es la campaña de denigración del niño contra un padre, una campaña que no tiene justificación”. Esta campaña de denigración de la que habla el SAP, conlleva la atribución de falsedad al testimonio de los menores, en el contexto de la disputa por la guarda y custodia o el régimen de visitas con éstos. Ello implica que, si el menor manifiesta que está siendo maltratado o abusado por uno de los progenitores, el SAP determina que su testimonio es falso y que concomitantemente se dejará de investigar el posible abuso o maltrato. Autores como Vilalta (2011) reafirman la existencia del SAP, pero como explican Clemente y Padilla (2015) "Vilalta, al igual que Gardner, ahonda en la perspectiva de basarse en intuiciones y dejar de lado los datos".

El SAP que Gardner formuló, se inspira en psicoanálisis de Freud: tanto uno como el otro son hipótesis no falsables, ya que tanto la aceptación como la no aceptación del diagnóstico suponen la afirmación de éste, en el SAP el menor miente cuando vilipendia al padre (normalmente el progenitor no custodio es el varón) y en psicoanálisis el menor fantasea cuando relata recuerdos de abuso sexual durante la infancia, según Gardner los niños pueden tener fuertes impulsos sexuales e iniciar ellos encuentros sexuales con los adultos mintiendo y culpando luego al adulto si la relación es descubierta (Gardner, 1986, 1992) y Freud a través de la teoría edípica, nos traslada que el niño es un seductor capaz de matar a su padre para usurpar su lugar en relación a su madre. En suma, tanto el SAP como el psicoanálisis, se adscriben al campo de la ideología huyendo del de la ciencia. Clemente y Padilla (2015) demuestran con un estudio que los menores tienden a decir la verdad, por lo que partiendo de ello, en el presente estudio se abordará si la edad es una variable mediadora en la veracidad del testimonio de los menores y en las posibilidades de manipulación de éste por parte de terceras personas. Se va a hipotetizar que la variable edad no influye ni en la veracidad del testimonio de los menores ni en su posible manipulación.

\section{Participantes}

\section{Método}

Se realiza un estudio en un colegio con 300 menores de ambos sexos y de edades comprendidas entre 6 y 12 años. 


\section{Instrumentos}

Mediante entrevista personal con cada niño se les pregunta sobre lo que han presenciado, consignándose los resultados en una hoja de respuesta por parte del equipo de investigación. La entrevista con cada niño es individual y al término de la misma, el niño no vuelve al aula donde esperan los que aún no han sido entrevistados para evitar la contaminación. Las variables dependientes van a ser los testimonios de los niños en las dos fases del experimento y las variables independientes serán el hecho de presenciar o no una agresión, la presión e imagen que se les proyecta a los niños sobre la parte actora de la agresión y la edad de los niños.

\section{Procedimiento.}

Tras los pertinentes permisos a la dirección de un centro de educación primaria, se les explica a profesores $\mathrm{y}$ tutores el experimento, quienes explican y piden permiso y discreción a los padres. El experimento va a constar de dos fases que se van a desarrollar en tres días diferentes, estando los niños divididos en dos grupos:

- El primer día, un grupo va a presenciar una agresión verbal simulada y el otro no.

- El segundo día, los dos grupos van a ser preguntados, en el primer caso, para que relaten que ha ocurrido y en el segundo, se les va a preguntar si han visto algo problemático. Se utilizó un diseño con medidas post.

- El tercer día, se les pregunta a los dos grupos de niños sobre lo que han visto, pero informándoles previamente, de que la persona actora de agresión va a ser o no su tutora en el futuro e infiriéndoles una imagen positiva o negativa de la misma, creándose por tanto cuatro posibles grupos de información. El diseño es factorial $\mathrm{A} * \mathrm{~B}, 2 * 2$, solo con medidas post.

La información de lo que los niños han presenciado o no, se recoge dos veces, tras lo cual, se les comunica a los niños que la agresión no ha sido real, y que su fin era ver como ellos reaccionarían.

\section{Resultados}

La edad de los menores no es una variable mediadora de la veracidad del testimonio de éstos. Los resultados del estudio revelan que los menores dicen la verdad en sus testimonios independientemente de sus edades. Dentro de ello, cabe señalar que, cuando los menores presencian la agresión, el porcentaje de testimonios veraces es muy elevado y similar en todas las edades estudiadas. Y cuando no presencian la agresión, los menores de 7, 8 y 9 años son los que más tienden a decir la verdad, y los de 10 años los que obtienen el menor porcentaje de veracidad. No es significativo estadísticamente en la veracidad del testimonio, el que los menores presencien la agresión o no, pero es en los niños de 10 años donde mayor peso tienen esta variable, $\mathrm{y}$ en los niños de 7 años donde no tienen ninguna repercusión.

Si a los menores que han presenciado la agresión, se les intenta manipular introduciendo elementos de presión e imagen, la veracidad del testimonio de éstos no varía estadísticamente de manera significativa. Se concluye, por tanto, que los menores no mienten es sus testimonios cuando presencian un hecho conflictivo, independientemente de sus edades.

Tabla 1. Información en función de la producción de una agresión verbal

\begin{tabular}{|c|c|c|c|c|c|c|c|c|c|c|c|c|c|c|}
\hline & & & \multicolumn{2}{|c|}{ Edad 1} & \multicolumn{2}{|c|}{ Edad 2} & \multicolumn{2}{|c|}{ Edad 3} & \multicolumn{2}{|c|}{ Edad 4} & \multicolumn{2}{|c|}{ Edad 5} & \multicolumn{2}{|c|}{ Edad 6} \\
\hline & & & Verdad & Mentira & Verdad & Mentira & Verdad & Mentira & Verdad & Mentira & Verdad & Mentira & Verdad & Mentira \\
\hline \multirow{6}{*}{$\begin{array}{l}\text { Tipo } \\
\text { agresion }\end{array}$} & Agresión & Frecuencia & 23 & 3 & 22 & 2 & 27 & 0 & 24 & 0 & 25 & 1 & 24 & 2 \\
\hline & & (N) & & & & & & & & & & & & \\
\hline & & Porcentaje & $88,5 \%$ & $11,5 \%$ & $91,7 \%$ & $8,3 \%$ & $100,0 \%$ &, $0 \%$ & $100,0 \%$ &, $0 \%$ & $96,2 \%$ & $3,8 \%$ & $92,3 \%$ & $7,7 \%$ \\
\hline & No & Frecuencia & 9 & 11 & 22 & 2 & 18 & 8 & 14 & 8 & 6 & 19 & 14 & 11 \\
\hline & agresión & $(\mathrm{N})$ & & & & & & & & & & & & \\
\hline & & Porcentaje & $45,0 \%$ & $55,0 \%$ & $91,7 \%$ & $8,3 \%$ & $69,2 \%$ & $30,8 \%$ & $63,6 \%$ & $36,4 \%$ & $24,0 \%$ & $76,0 \%$ & $56,0 \%$ & $44,0 \%$ \\
\hline \multirow[t]{2}{*}{ Total } & & Frecuencia & 32 & 14 & 44 & 4 & 45 & 8 & 38 & 8 & 31 & 20 & 38 & 13 \\
\hline & & Porcentaje & $69,6 \%$ & $30,4 \%$ & $91,7 \%$ & $8,3 \%$ & $84,9 \%$ & $15,1 \%$ & $82,6 \%$ & $17,4 \%$ & $60,8 \%$ & $39,2 \%$ & $74,5 \%$ & $25,5 \%$ \\
\hline
\end{tabular}


Tabla 2. Efectos del grado de presión y de la imagen para los sujetos que presenciaron la agresión

\begin{tabular}{|c|c|c|c|c|c|c|c|c|c|c|c|c|c|c|c|}
\hline \multicolumn{16}{|c|}{ Tabla de contingencia Información * Presión * } \\
\hline \multirow[t]{3}{*}{ Fase 2} & & & & & & \multicolumn{2}{|c|}{ Presión Edad } & \multicolumn{2}{|c|}{ Presión Edad } & \multirow{2}{*}{\multicolumn{2}{|c|}{$\begin{array}{c}\text { Presión Edad } \\
4 \\
\end{array}$}} & \multicolumn{2}{|c|}{ Presión Edad 5} & \multicolumn{2}{|c|}{ Presión Edad 6} \\
\hline & & & & \multicolumn{2}{|c|}{ Presión Edad 1} & 2 & & & 3 & & & & & & \\
\hline & & & & Alta & Baja & Alta & Baja & Alta & Baja & Alta & Baja & Alta & Baja & Alta & Baja \\
\hline \multirow[t]{9}{*}{ Verdad } & Información & Buena & Frecuencia & 6 & 5 & 10 & 1 & 6 & 7 & 6 & 6 & 6 & 6 & 5 & 6 \\
\hline & & persona & $(\mathrm{N})$ & & & & & & & & & & & & \\
\hline & & & Porcentaje & $54,5 \%$ & $45,5 \%$ & $90,9 \%$ & $9,1 \%$ & $46,2 \%$ & $53,8 \%$ & $50,0 \%$ & $50,0 \%$ & $50,0 \%$ & $50,0 \%$ & $45,5 \%$ & $54,5 \%$ \\
\hline & & Mala & Frecuencia & 6 & 5 & 11 & 1 & 7 & 7 & 6 & 6 & 6 & 7 & 7 & 6 \\
\hline & & persona & (N) & & & & & & & & & & & & \\
\hline & & & Porcentaje & $54,5 \%$ & $45,5 \%$ & $91,7 \%$ & $8,3 \%$ & $50,0 \%$ & $50,0 \%$ & $50,0 \%$ & $50,0 \%$ & $46,2 \%$ & $53,8 \%$ & $53,8 \%$ & $46,2 \%$ \\
\hline & Total & & Frecuencia & 12 & 10 & 21 & 2 & 13 & 14 & 12 & 12 & 12 & 13 & 12 & 12 \\
\hline & & & $(\mathrm{N})$ & & & & & & & & & & & & \\
\hline & & & Porcentaje & $54,5 \%$ & $45,5 \%$ & $91,3 \%$ & $8,7 \%$ & $48,1 \%$ & $51,9 \%$ & $50,0 \%$ & $50,0 \%$ & $48,0 \%$ & $52,0 \%$ & $50,0 \%$ & $50,0 \%$ \\
\hline \multirow[t]{8}{*}{ Mentira } & Información & Buena & Frecuencia & 0 & 1 & 1 & & & & & & 1 & & 1 & 0 \\
\hline & & persona & $(\mathrm{N})$ & & & & & & & & & & & & \\
\hline & & & Porcentaje &, $0 \%$ & $100,0 \%$ & $100,0 \%$ & & & & & & $100,0 \%$ & & $100,0 \%$ &, $0 \%$ \\
\hline & & Mala & Frecuencia & 1 & 2 & 1 & & & & & & 1 & & 0 & 1 \\
\hline & & persona & $(\mathrm{N})$ & & & & & & & & & & & & \\
\hline & & & Porcentaje & $33,3 \%$ & $66,7 \%$ & $100,0 \%$ & & & & & & $100,0 \%$ & &, $0 \%$ & $100,0 \%$ \\
\hline & Total & & $\begin{array}{l}\text { Frecuencia } \\
\text { (N) }\end{array}$ & 1 & 3 & & & & & & & & & 1 & 1 \\
\hline & & & Porcentaje & $25,0 \%$ & $75,0 \%$ & & & & & & & & & $50,0 \%$ & $50.0 \%$ \\
\hline
\end{tabular}

Tabla 3. Efectos del grado de presión y de la imagen para los sujetos que no presenciaron la agresión

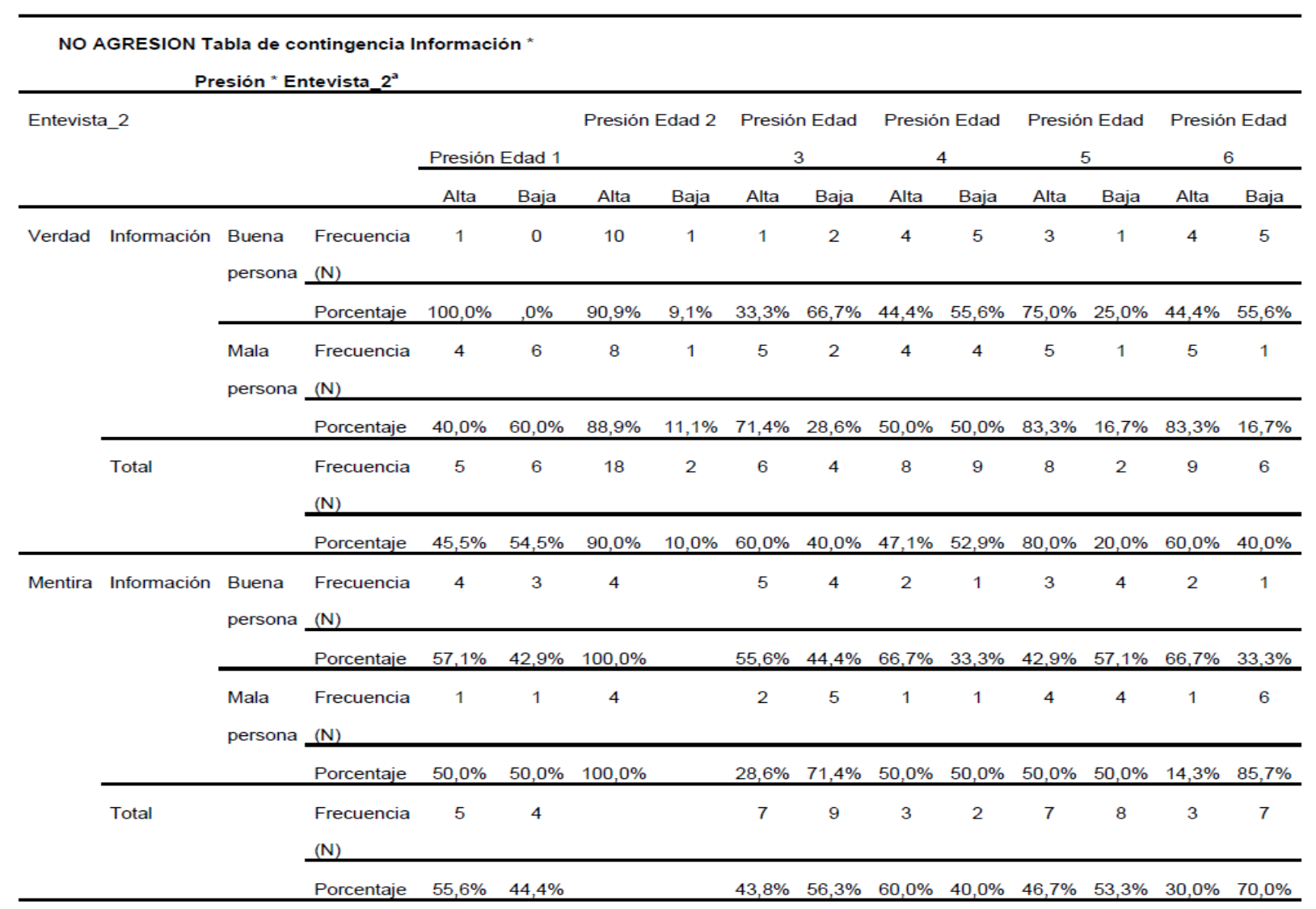




\section{Discusión}

Los menores tienden a decir la verdad, independientemente de la edad. En el caso de que presencien algo conflictivo, dicen la verdad en un porcentaje amplísimo y similar en todas las edades comprendidas en este estudio. $\mathrm{Y}$ al introducir las variables de presión e imagen el testimonio de los menores prácticamente no sufre variación. Y cuando no presencian la agresión, los menores de 7, 8 y 9 años son los que más tienden a decir la verdad, y los de 10 años los que obtienen el menor porcentaje de veracidad. No es significativo estadísticamente en la veracidad del testimonio, el que los menores presencien la agresión o no, pero es en los niños de 10 años donde mayor peso tienen esta variable, y en los niños de 7 años donde no tienen ninguna repercusión. Cuando no presencian ningún episodio conflictivo, pero se les infiere una pregunta sugestiva a cerca de lo que han presenciado, los menores del presente estudio, en un $40 \%$ fabrican un recuerdo. Este recuerdo es compatible con un fenómeno presente en el funcionamiento de la memoria de todo ser humano y que no constituye ninguna patología: la falsa memoria. El concepto de la falsa memoria se introdujo en la psicología tras las investigaciones de Loftus, Miller y Burns (1978). Si a los menores se les intenta manipular introduciendo elementos de presión e imagen, la veracidad del testimonio de éstos no varía significativamente, Se concluye, por tanto, que los menores no mienten es sus testimonios independientemente de sus edades.

Se demuestra que las ideas de Gardner no tienen soporte empírico, el presente estudio demuestra lo contrario. Uno de los axiomas del SAP es que el niño es mentiroso por naturaleza y que es capaz de sostener una mentira con tal de perjudicar a un tercero (el progenitor no custodio), sin embargo los datos demuestran que los menores, en los distintos tramos de edad, objetos del estudio, dicen la verdad cuando presencian un hecho y pueden fabricar un recuerdo cuando no lo presencian, como el resto de seres humanos. Y este recuerdo fabricado, gira en torno a la interacción de los menores entre ellos mismos o entre ellos y la tarea o actividad, no en el lanzamiento de acusaciones de hechos conflictivos atribuidos a terceras personas. El menor pues, no es mentiroso por naturaleza. Al autodefinirse como síndrome, el SAP lo hace alegando que en la campaña de denigración que el menor hace contra el progenitor no custodio, están presentes además de la manipulación del progenitor con el que el menor convive, las propias fabricaciones del menor. Asentado ya que el menor no miente, los resultados del estudio, demuestran además que la premisa de la facilidad de la manipulación sobre los menores también es falsa. El testimonio de los menores, no se ve alterado cuando a éstos se les plantea la posibilidad de dependencia, en una relación de asimetría, de la persona actora de la agresión y además de les proyecta una determinada imagen de ésta. La introducción de estas variables de presión e imagen en el diseño no es baladí: recrean por un lado, la situación de asimetría que se da entre el menor y el progenitor con el que convive y al que Gardner atribuye la manipulación sobre el menor y la imagen negativa que supuestamente este progenitor infiere al menor sobre el progenitor no custodio. Por tanto, esa falsa memoria, presente en los menores en los tramos de edad sujetos a estudio, no está sujeta a variables de presión e imagen, como preconiza el SAP.

Los resultados del presente estudio refutan una de las ideas principales del SAP que Gardner propuso, la de que los niños mienten y son fácilmente manipulables, capaces además de mantener una mentira con el fin de perjudicar a un tercero y verifican en contraposición, las teorías de autores como Clemente (2013), quien afirma que "el SAP es un atentado contra la ciencia, contra el Estado de Derecho, y contra los menores y sus progenitores. De hecho, una teoría no científica no debería ser utilizada dentro del sistema de administración de Justicia, ya que en ese caso el primer perjudicado será el menor" y Escudero et al. (2010, p. 22) quienes sostienen que "no existe dicha acreditación sobre la metodología, y el constructor del supuesto PAS no puede garantizar en modo alguno la falsedad o no de las denuncias; si pueden existir múltiples explicaciones válidas sobre el rechazo del menor hacia el progenitor las cuales el supuesto PAS no pueda descartar; si la posibilidad de falsos positivos (determinar erróneamente que un menor tiene un supuesto PAS sin tenerlo) es tan elevado que en tal caso la medidas de cambio de custodia pueden suponer realmente un riesgo y daño inasumible para el menor; y si pudiéndose demostrar que los fundamentos del supuesto PAS se han construido falseando la terminología empleada y establecido una lógica que permita justificar cualquier resultado a priori, ¿cabría entonces que un sistema de protección como es el judicial permitiese mantener su uso?". Escudero et al. (2008) considera por todo ello que el sistema judicial debe revisar el empleo del PAS y sus medidas, que bajo el reclamo de «terapéuticas» sólo pueden generar daño psíquico y perversión del uso de la ciencia.

Como conclusión final, decir que el sistema legal ha de tomar en su debida consideración el testimonio de los menores, pues se demuestra que son testimonios veraces, sujetos a las mismas dinámicas de los testimonios de los adultos en cuanto a lo que a la falsa memoria se refiere, sin poder atribuir al testimonio de los menores varones y mujeres la falsedad y animosidad que el SAP categóricamente les atribuye.

Como limitaciones más destacadas al presente estudio destacar, que se ha llevado a cabo con una muestra de niños que no están sometidos a la situación conflictiva en la que se desarrolla el concepto del SAP que se ha estudiado, y ello por razones de tamaño de la muestra necesaria para que arrojase resultados estadísticos significativos. Otra limitación sería que el estudio se ha hecho en un rango de edades desde los 6 a los 12 años y en la realidad, bajo los criterios del SAP, la veracidad del testimonio de los menores está en entredicho desde que se disuelve la unidad familiar hasta que el menor alcanza la mayoría de edad. Por último la muestra sobre la que se hace el presente estudio ha podido presenciar una agresión verbal, que por motivos éticos no llega a ser de 
agresión física o sexual, como pueden ser presenciadas por los menores en la realidad.

\section{Referencias bibliográficas}

Clemente, M. (2013). El Síndrome de alienación parental: un atentado contra la ciencia, contra el estado de derecho, y contra los menores y sus progenitores. Infancia, Juventud y Ley, 4, 48-57.

Clemente, M. (2014). Aproximaciones psicológicas y jurídicas a la guarda y custodia. Madrid: Síntesis.

Clemente, M. \& Padilla-Racero, D. (2015). Are children susceptible to manipulation? The best interest of children and their testimony. Children and Youth Services Review, 51, 101-107. DOI: 10.1016/j.childyouth.2015.02.003.

Escudero et al. (2008). La Lógica del Síndrome de Alienación Parental de Gardner (SAP): Del Síndrome "Puro" a la "Terapia de la Amenaza". Revista de la Asociación Española de Neuropsiquiatría, 28 (2), 285 307.

Escudero. A.; González, D.; Méndez, R.; Naredo, C.; Pleguezuelos, E.; Vaccaro, S. \& Pérez, A.M. (2010).
Informe del grupo de trabajo de investigación sobre el llamado síndrome de alienación parental. Madrid: Ministerio de Sanidad, Política Social e Igualdad.

Gardner R. (1985). Recent trends in divorce and custody litigation. Academy Forum, 29, 3-7.

Gardner, R.A. (1986). Child Custody Litigation: A Guide for Parentsand Mental Health Professionals. Cresskill, NJ: Creative Therapeutics.

Gardner, R. A. (1991). Legal and Psychotherapeutic Approaches to the Three Types of Parental Alienation Syndrome Families. When Psychiatry and the Law Join Forces. Court Review, 28 (1), pp. 14-21.

Gardner, R.A. (1992). True and False Accusations of Child Sex Abuse. Cresskill, NJ: Creative Therapeutics

Loftus, E. F., Miller, D. G. \& Burns, H. J. (1978). Semantic integration of verbal information into a visual memory. Human Learning and Memory, 4, 19-31.

Vilalta, R.J. (2011). Descripción del Síndrome de Alienación Parental en una muestra forense. Psicothema, 23 (4), 636-641. 